\title{
Study on the design of semiconducting anti icing RTV coatings
}

\author{
Fang Zhenyu1, a, Wu Peiyun2, b \\ 1State Grid Liaoning Electric Power Company Anshan Power Supply \\ Company, Liaoning, Anshan, 114000, China \\ 2State Grid Liaoning Electric Power Company Anshan Power Supply \\ Company, Liaoning, Anshan, 114000, China \\ azy_fang0432@163.com,b154371977@qq.com
}

\begin{abstract}
At present, the application of transmission line insulator anti pollution flashover coating is mainly the room temperature vulcanized (Room Temperature Vulcanized, RTV) silicone rubber coating. Insulator coated with RTV paint, not only enhanced the hydrophobicity on the surface of the insulator, pollution layer of its surface area poly also obtains the hydrophobicity of different degree, namely the coating has good hydrophobicity transfer. The hydrophobic coating with excellent surface water is less, not to form water film continuously, however when the coating is covered with ice after the loss of hydrophobicity, and ordinary as the porcelain insulator. Based on this, this paper puts forward to want to have certain characteristics in basic water repellent anti icing effect, the silicone rubber coating modified into a semiconductor coating, which has two characteristics of hydrophobic properties and semi conductive properties, direct coated ice covering proof coating in porcelain insulator surface can be. Hydrophobic properties can reduce the adhesion surface ice and materials and reduce the density of ice coating, and the changes of ice form, but not to delay and prevent icing. The volume resistivity of coating is the main factor affecting the overall amount of insulator fever. Through the adjustment of the volume resistivity of coating can make the insulator to obtain large.
\end{abstract}

Keywords: anti icing, semiconductor RTV, hydrophobic anti icing

\section{Introduction}

In recent years, transmission lines all over the world have suffered harm caused by icing. The relative power system encounters other natural disasters, ice climate continues to often bring more serious consequences, light ice flashover occurs, heavy then lead to tower collapse, line breaking, even network paralysis. How to effectively reduce the occurrence of ice flashover of insulator, prevent the insulator icicle bridging, improve the insulator flashover voltage, these have become a problem urgent to solve.

The harm of transmission line icing mainly include: 
(1) Iced Insulator String adjacent umbrella disk will be the ice bridge, the leakage distance of insulator is greatly shortened, and the ice water conductivity is relatively high, extremely easy to cause the ice flashover.

(2) Transmission line and tower icing, exceeding the design limits of mechanical bearing ability, cause disconnection tower collapse accident.

(3) Conductor icing, wire and wind resistance becomes larger, and the uneven Ice Shedding caused by wire jump, resulting in inter phase short circuit fault.

At present, the application of transmission line insulator anti pollution flashover coating is mainly the room temperature vulcanized (Room Temperature Vulcanized, RTV) silicone rubber coating. Insulator coated with RTV paint, not only enhanced the hydrophobicity on the surface of the insulator, pollution layer of its surface area poly also obtains the hydrophobicity of different degree, namely the coating has good hydrophobicity transfer [80]. The hydrophobic coating with excellent surface water is less, not to form water film continuously, however when the coating is covered with ice after the loss of hydrophobicity, and ordinary as the porcelain insulator. Use only the hydrophobic anti icing work is hard, therefore, this paper puts forward to want to have certain characteristics in the basis of water repellent anti icing effect, the silicone rubber coating modified into a semiconductor coating. Will this paint coated on the surface of the insulator coating after curing, prevent the formation of ice coated insulator coating. The coating has excellent hydrophobic properties and semi conductive properties.

\section{Icing prevention mechanism of semiconductor RTV coating}

The anti icing mechanism of semiconductor RTV coating is as shown in Fig.1.

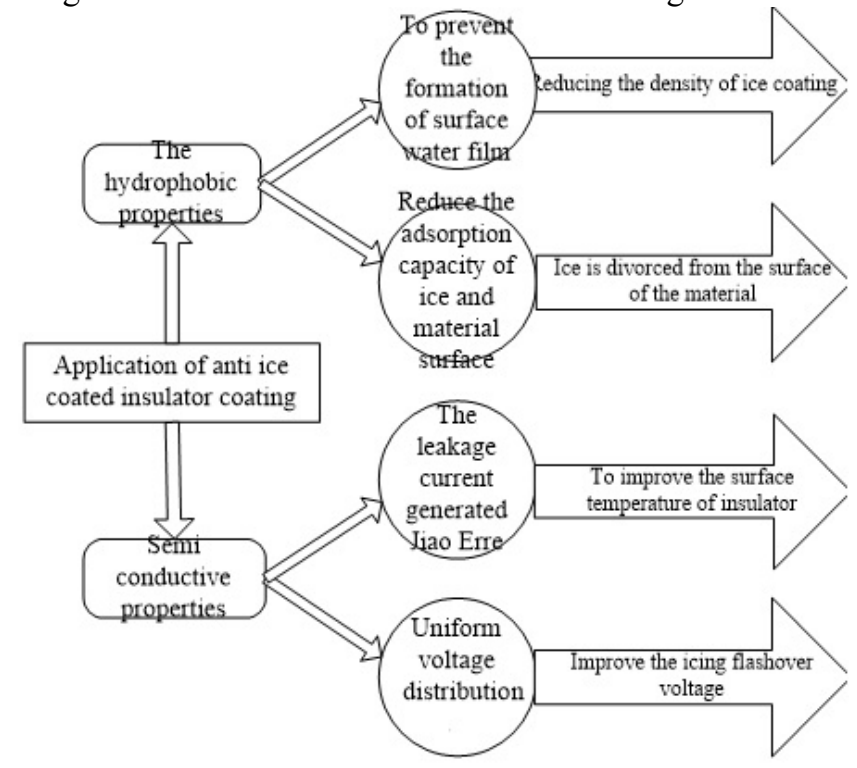


Fig.1. Icing prevention mechanism of semiconductor RTV coating

Semiconductor silicon rubber is hydrophobic hydrophobic stronger, more strong, the adhesion between the coating surface and ice is smaller, the insulator icing off easily. Water in the form of existence is not a continuous film of water surface hydrophobicity of small water droplets, but independent of, and most of the landing fall by itself in the coating surface water droplets will, greatly reducing the amount of ice coating. Residues at the surface of the water drops below zero will freeze a small ice, ice piled up to form ice, but between these small ice exists large air gaps, so the formation of ice density is far better than the water film formed ice density must be small. To reduce the ice density will also reduce the amount of ice coating on the surface of the coating.

The formed coating semiconductor silicon rubber with conductive properties, but will not damage the insulator insulating, semiconducting properties so that the coating anti icing effect in improving. To improve the surface leakage current Joule heat generated by the current increase in surface heat, improve the surface temperature. Surface temperature raise and delaying the formation of ice covered the surface, if the surface temperature can be stabilized above zero, the formation will be able to completely prevent ice. The semi conducting coating also makes the voltage distribution of insulator string on more evenly, reducing the probability of flashover occurrence.

\section{Heating mechanism of coating semiconductor RTV insulators}

Application of semiconductor RTV coated insulators, because the coating with semi conductive properties, thus reducing the insulation resistance between the two poles of the ceramic insulator, when electricity runs through the coating leakage current causes Joule heat, improve the surface temperature of the insulator. Ceramic dielectric insulator itself will produce dielectric loss heating is also in strong electric field. In the guarantee under the condition of insulator insulating performance, improve the insulator heating is conducive to the formation of surface to prevent icing.

Insulator gross calorific value $Q$ is mainly composed of four parts, can be expressed by the following equation:

$$
Q=Q_{C}+Q_{I}+Q_{S}+Q_{T}
$$

(1)

Among them:

$Q_{C}$ Heat generated by dielectric loss.

$Q_{I}$ _ The porcelain body internal penetration leakage current caused by fever.

$Q_{S} \ldots$ The heating surface creepage caused by leakage current.

$Q_{T}$ Heat coating, the Joule heat is mainly generated by leakage current.

Dielectric loss heating refers to the power loss of any dielectric are under 
pressure, including dipole polarization, polarization loss caused by the interlayer polarization, conductance and conductance with a dielectric loss caused by the. Dielectric loss heat insulator can be calculated using the formula:

$$
Q_{C}=U^{2} \omega C_{o} \tan \delta
$$

Where $U$ is the insulator voltage between electrodes, $\omega$ as the operating

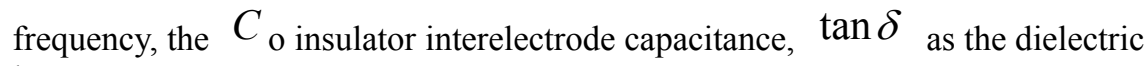
loss tangent.

For the ceramic insulator, dielectric loss angle tangent $\tan \delta$ is about $0.015 \sim$ 0.025 , take the ceramic insulator interelectrode capacitance is $C=50 \mathrm{pF}$ Dielectric loss heating normal insulator is relatively small, and the dielectric loss of insulator running is basically unchanged, can improve the heating amount of coating makes the surface temperature rise of insulator. If it can be improved by adjusting the recipe of paint coating of heat, can keep the surface temperature of the insulator is above zero, delay or prevent the formation of ice.

Insulator string of monolithic insulator simplified equivalent circuit as shown in Fig.2. The Communist Party of China have insulator insulator strings n, where in the $\mathrm{I}$ insulator interelectrode capacitance is $C_{i}$, assuming a $C_{1}=C_{2}=\cdots \cdots=C_{n}=C_{o}$ 。 $R_{X}$ insulator equivalent resistance.

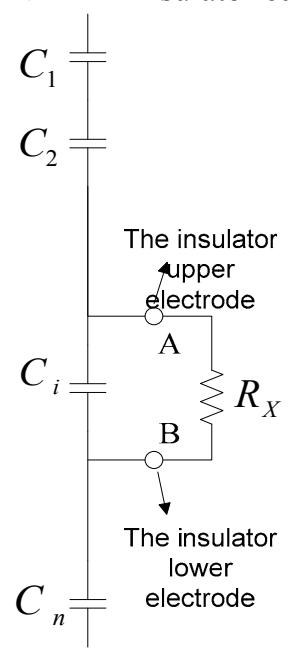

Fig.2. A simplified equivalent circuit for chip insulator Based on Thevenin's theorem, if open A, B, B between the two, voltage A for the I insulator on the interelectrode voltage $U_{i}$. When $\mathrm{A}, \mathrm{B}$ two short circuit impedance, A, B for n-1 the remaining interelectrode capacitance $C_{0}$ series with $C_{i}$ parallel, the equivalent capacitance of 
$C_{D}=C_{i}+\frac{C_{o}}{n-1}=C_{o}+\frac{C_{o}}{n-1}=\frac{n}{n-1} C_{o}$

Fig. 3 can be further simplified into a simple equivalent circuit of monolithic insulator in Fig.3:

Fig.3. A simplified equivalent circuit for chip insulator

$R_{X} \quad$ as the equivalent resistance insulator, $X_{D}$ for the equivalent capacitance, $X_{D}=j \omega C_{D}=\frac{n}{n-1} j \omega C_{o}$ 。

$$
P=U_{i}^{2} R_{X} /\left(X_{D}^{2}+R_{X}^{2}\right)=U_{i}^{2} /\left(\frac{X_{D}^{2}}{R_{X}}+R_{X}\right)
$$
The $X_{D}$ is the equivalent capacitance. About $R_{X}$ of the $\frac{X_{D}^{2}}{R_{X}}+R_{X}$
derivative:

Make

$$
d\left(\frac{X_{D}^{2}}{R_{X}}+R_{X}\right) / d R_{X}=-\frac{X_{D}^{2}}{R_{X}^{2}}+1=0,
$$

There are $R_{X}=X_{D}$. When the $R_{X}=X_{D}$, which is when the insulation resistance insulator is equal to the equivalent capacitance value, the heating power insulator $P$ and reached a maximum value.

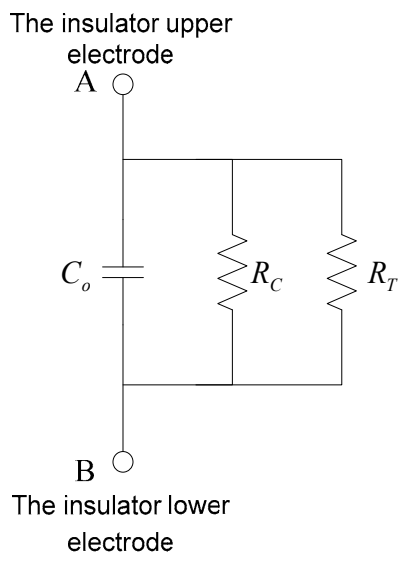

Fig.4. A simplified equivalent circuit for chip insulator with coatings 
When the surface of the insulator is coated with a semiconductor coating, equivalent to the parallel equivalent resistance of a coating, the other $R_{C}$ is the equivalent resistance of the medium loss. The equivalent resistance insulator for coating $R_{X}$ equivalent resistance and equivalent resistance of $R_{T}$ parallel dielectric loss $R_{C}$. After applying the coating of monolithic insulator equivalent circuit as shown in Fig.4:

Available:

$$
\frac{1}{R_{X}} \approx \frac{1}{R_{C}}+\frac{1}{R_{T}}
$$

The dielectric loss equivalent resistance of $R_{C}=1 / \omega C_{0} \tan \delta$, normal $R_{C}$ ceramic insulator is about $2000 \sim 4000 M \Omega$.

When porcelain insulator surface coating clean anti icing silicone rubber coated with semiconductor properties, dielectric loss equivalent resistance of $R_{C}$ is much greater than $R_{T}$, so $R_{X} \approx R_{T}$. At this moment, insulator heating concentrated in the disk surface, which improved the insulator surface temperature. QT is greater than QC. For a complete porcelain body, clean surface coating insulators, QI and QS is much lower than that of QT. So the total calorific value $Q \approx Q_{T}$ 。 The equivalent resistance of the coating is determined by the volume resistivity of the coating, directly determines the size of QT. Therefore in the coated with ice covering proof coating, the volume resistivity of coating are the main factors that influence the total quantity of heat insulator Q.

\section{Conclusion and prospect}

This paper presents the anti icing mechanism of semiconductor coating, which has two characteristics of hydrophobic properties and semi conductive properties, direct coated ice covering proof coating in porcelain insulator surface can be. Hydrophobic properties can reduce the adhesion surface ice and materials and reduce the density of ice coating, and the changes of ice form, but not to delay and prevent icing. The volume resistivity of coating is the main factor affecting the overall amount of insulator fever. Through the adjustment of the volume resistivity of coating can make the insulator to obtain large.

The anti icing effect of semiconductor silicon rubber is mainly reflected in the electrothermal effect. Through adjusting the proportion and distribution of conductive filler in semiconductor silicon rubber inside, can for the volume resistivity of semiconductor silicon rubber were adjusted, makes the insulator surface in coated semiconductor silicon rubber and fully cured, insulator leakage 
current is controlled in a certain range, thereby generating electric effect better. If the leakage current is smaller, will because of the weaker thermal effect it brings rather than as an anti icing effect accordingly, but in the case of leakage current is large, will to power system bring very serious power loss, so need through the volume resistance regulating semiconductor silicon rubber leakage current flowing through the rate of change of the size of the surface of the insulator find the critical value, the leakage current, which not only can satisfy the need of anti icing aspect, but also the power loss in the lower control level. In order to obtain the critical values of the leakage current, the electric heating effect on the insulator surface are evaluated in the further work.

\section{References}

[1] Zhang Hongxian, Lu Jiazheng, Peng Jiwen, etc. Analysis of 2008 2009 in Hunan power grid ice statistics [J]. Hunan electric power, 2009 (05).

[2] Lu Hangbo. Preliminary analysis of 2008 in North Guangxi overhead transmission line icing [J]. Red water river, 2009 (02).

[3] Zhang Aihu. The analysis of tower 45 inverted tower icing which is in the 220kV Xun Miao II line / Xun Xi lin. [J]. Jiangxi electric power, 2008

[4] Zhao Lijin, Wu Xiangqian, Li Wei, etc. Analysis of Guizhou power grid in 2008 ice disaster accident and its countermeasures [C]. Chinese Guizhou Guiyang: 2008

[5] Li Qiang in 2008. Freezing rain and snow disaster analysis and Enlightenment of power network [J]. electric power construction, 2008 (06).

[6] Deng Chenggang. The 2008 Guizhou grid damage reason analysis and thinking [C]. China Guizhou Guiyang: 2008

[7] Chen Song. Prevention and treatment measures of transmission line icing in Luzhou area [J]. Sichuan Electric Power Technology, 2008

[8] Chen Yuan, Zhang Zhangkui, Liu Juan, etc. Beijing Tianjin Tangshan power grid transmission line icing tripping analysis $[\mathrm{J}]$. North China Electric Power, 2003 (04).

[9] Jia Z, Gao H, Wang L, et al. Study on the Hydrophobic Transfer Based on 
the Theory of Absorption and Cohesion[J]. IEEE Intern. Conf. Properties and Applications of Dielectric Materials, 2003(162 166).

[10] Hu Shizheng. Heating mechanism and thermal image characteristics of faulty insulator [J]. Grid technology, 1997 (10). 